\title{
Gaussian Mersenne numbers and generalized Mersenne quaternions
}

\author{
Ahmet Daşdemir ${ }^{1}$ and Göksal Bilgici ${ }^{2}$ \\ ${ }^{1}$ Department of Mathematics, Faculty of Arts and Sciences, Kastamonu University \\ Kuzeykent Campus, 37150 Kastamonu, Turkey \\ e-mail: ahmetdasdemir37@gmail.com \\ ${ }^{2}$ Department of the Computer Education and Instructional Technologies, Education Faculty, \\ Kastamonu University 37100, Kastamonu, Turkey \\ e-mail: gbilgici@kastamonu.edu.tr
}

Received: 14 September 2018

Revised: 26 April 2019

Accepted: 1 May 2019

\begin{abstract}
In this study, we introduce a new class of quaternions associated with the well-known Mersenne numbers. There are many studies about the quaternions with special integer sequences and their generalizations. All of these studies used consecutive elements of the considered sequences. Here, we extend the usual definitions into a wider structure by using arbitrary Mersenne numbers. Moreover, we present Gaussian Mersenne numbers. In addition, we give some properties of this type of quaternions and Gaussian Mersenne numbers, including generating function and Binet-like formula.
\end{abstract}

Keywords: Mersenne quaternions, Generating function, Binet's formula, Gaussian Mersenne numbers, Catalan's identity.

2010 Mathematics Subject Classification: 11B37, 16H05, 11 R52.

\section{Introduction}

In 1843, the Irish mathematician Sir William Rowan Hamilton introduced quaternions which are members of a non-commutative division algebra in 1843 as a kind of extension of complex numbers to higher spatial dimensions. Let $\mathrm{R}^{r, s}$ be the vector space with quadratic forms and $C l_{r, s}(\mathrm{R})$ be the Clifford algebra on $\mathrm{R}^{r, s}$. This means that a standard basis $\left\{e_{i}\right\}$ has $r$ vectors 
square to 1 and $s$ vectors square to -1 . Quaternions can be constructed as the even subalgebra of the $C l_{0,3}(\mathrm{R})$. For more details, the monograph [4] can be investigated.

A quaternion $q$ is formally defined as follows:

$$
q=q_{0}+i q_{1}+j q_{2}+k q_{3}
$$

where $q_{0}, q_{1}, q_{2}$, and $q_{3}$ are any real numbers, and the standard basis $\{1, i, j, k\}$ satisfies

$$
i^{2}=j^{2}=k^{2}=-1, i j=-j i=k, j k=-k j=i, k i=-i k=j .
$$

The conjugate of $q$ is

$$
q^{*}=q_{0}-i q_{1}-j q_{2}-k q_{3}
$$

and its norm is

$$
N(q)=q q^{*}=q_{0}^{2}+q_{1}^{2}+q_{2}^{2}+q_{3}^{2} .
$$

In [5], Horadam used another perspective to the above-mentioned subject by defining the Fibonacci and Lucas quaternions in the forms

$$
Q_{n}=F_{n}+F_{n+1} e_{1}+F_{n+2} e_{2}+F_{n+3} e_{3}
$$

and

$$
P_{n}=L_{n}+L_{n+1} e_{1}+L_{n+2} e_{2}+L_{n+3} e_{3},
$$

respectively, where $F_{n}$ is the famous $n$th Fibonacci number and $L_{n}$ is the $n$-th Lucas number. In $[3,6]$, the Pell and Pell-Lucas quaternions are introduced as

$$
Q P_{n}=P_{n}+i P_{n+1}+j P_{n+2}+k P_{n+3}
$$

and

$$
Q P L_{n}=P L_{n}+i P L_{n+1}+j P L_{n+2}+k P L_{n+3},
$$

where $P_{n}$ and $P L_{n}$ are the $n$-th Pell and Pell-Lucas numbers, respectively. Szynal-Liana and Wloch [7] defined Jacobsthal and Jacobsthal-Lucas quaternions as

$$
J Q_{n}=J Q_{n}+i J Q_{n+1}+j J Q_{n+2}+k J Q_{n+3}
$$

and

$$
J L Q_{n}=J L Q_{n}+i J L Q_{n+1}+j J L Q_{n+2}+k J L Q_{n+3},
$$

where $J Q_{n}$ and $J L Q_{n}$ are the $n$-th Jacobsthal and Jacobsthal-Lucas numbers, respectively. There are many papers devoted to the quaternions whose coefficients are some special integer sequences, which are not mentioned here.

Based on these studies, we define a new class of quaternions and Gaussian Mersenne numbers associated with the Mersenne numbers. In addition, we give a Binet-like formula, generating functions, and certain identities such as Catalan's identity for these quaternions.

Before giving the main results, let us introduce these numbers briefly. Mersenne numbers are of the form

$$
M_{n}=2^{n}-1 .
$$


The first terms of Mersenne numbers are $1,3,7,15,31,63, \ldots$. These numbers are $(1)_{2},(11)_{2}$, $(111)_{2},(1111)_{2},(11111)_{2},(111111)_{2}, \ldots$ in binary system, respectively. They correspond to Cunningham numbers $C^{-}(2, n)$, which are numbers of the binomial number form $C^{-}(b, n)=$ $b^{n} \pm 1$. In order for $M_{n}$ to be a prime, $n$ must also be a prime. A Mersenne prime is a prime number, which is of the form $2^{n}-1$. There are 49 Mersenne primes that have been known so far. We refer to [8] for those who want to read the history of these interesting numbers. In [2], Catarino et al. extended Binet-like representation of the Mersenne numbers to the recurrence relation

$$
M_{n+1}=2 M_{n}+1
$$

or in the homogeneous form

$$
M_{n+2}=3 M_{n+1}-2 M_{n}
$$

where $M_{0}=0$ and $M_{1}=1$.

\section{Main results}

In this section, our definitions, some concepts, and the fundamental results are presented. First of all, we give the following definition.

Definition 2.1. For an integer $n$, the generalized Mersenne quaternion $\mathcal{M}_{n}^{(p, r, s)}$ is defined by

$$
\mathcal{M}_{n}^{(p, r, s)}=M_{n}+i M_{n+p}+j M_{n+r}+k M_{n+s}
$$

where $p, r$, and s are any integers.

We call $\mathcal{M}_{n}^{(1,2,3)}$ a Mersenne quaternion.

According to our definition, we have the following special cases:

- For $p=r=s=-n$, the usual Mersenne numbers are obtained:

$$
\mathcal{M}_{n}^{(-n,-n,-n)}=M_{n}
$$

- For $r=s=-n$, the generalized Gaussian Mersenne numbers are obtained:

$$
\mathcal{M}_{n}^{(p,-n,-n)}=M_{n}+i M_{n+p}
$$

- For $p=1$ and $r=s=-n$, the Gaussian Mersenne numbers are obtained:

$$
\mathcal{M}_{n}^{(1,-n,-n)}=M_{n}+i M_{n+1}
$$

- For $p=1, r=2$ and $s=3$, the Mersenne quaternions in the usual form of quaternions are obtained:

$$
\mathcal{M}_{n}^{(1,2,3)}=M_{n}+i M_{n+1}+j M_{n+2}+k M_{n+3}
$$


In [5], Horadam introduced the complex Fibonacci and Lucas numbers. These were named Gaussian Fibonacci and Lucas numbers by Berzsenyi [1] and they have been generally referred to this name since that day. So we prefer to use it for the second and third sequences. It should be noted that all the results obtained for the generalized Mersenne quaternions can be reduced to these numbers. To reduce the volume of the current paper, we only take the generalized Mersenne quaternions into account.

Generalized Mersenne quaternions satisfy the recurrence relations given in the following theorem.

Theorem 2.2. For any nonnegative integer $n$, we have

$$
\mathcal{M}_{n+1}^{(p, r, s)}=2 \mathcal{M}_{n}^{(p, r, s)}+\underline{\boldsymbol{A}}
$$

and

$$
\mathcal{M}_{n+1}^{(p, r, s)}=3 \mathcal{M}_{n}^{(p, r, s)}-2 \mathcal{M}_{n-1}^{(p, r, s)}
$$

where $\underline{\boldsymbol{A}}=1+i+j+k$.

Proof. Considering the Definition 2.1 and Eq. (3), we get

$$
\begin{aligned}
2 \mathcal{M}_{n}^{(p, r, s)} & =2 M_{n}+i\left(2 M_{n+p}\right)+j\left(2 M_{n+r}\right)+k\left(2 M_{n+s}\right) \\
& =M_{n+1}-1+i\left(M_{n+p+1}-1\right)+j\left(M_{n+r+1}-1\right)+k\left(M_{n+s+1}-1\right) \\
& =M_{n+1}+i M_{n+p+1}+j M_{n+r+1}+k M_{n+s+1}-(1+i+j+k) .
\end{aligned}
$$

The last equation gives Eq. (5). Using the Definition 2.1 and Eq. (4), Eq. (6) can be proved similarly.

It should be noted that Eq. (6) may also be represented by the matrix recurrence relation as follows:

$$
\mathbf{M}_{n+1}=\mathbf{R M}_{n}
$$

where

$$
\mathbf{M}_{n}=\left[\begin{array}{ll}
\mathcal{M}_{n+1}^{(p, r, s)} & \mathcal{M}_{n}^{(p, r, s)} \\
\mathcal{M}_{n}^{(p, r, s)} & \mathcal{M}_{n-1}^{(p, r, s)}
\end{array}\right]
$$

and

$$
\mathbf{R}=\left[\begin{array}{rr}
3 & -2 \\
1 & 0
\end{array}\right]
$$

If we extend Eq. (7) to the left to deal with terms of quaternions with large subscripts simultaneously, then we have

$$
\mathbf{M}_{n}=\mathbf{R}^{n-1} \mathbf{M}_{1}=\mathbf{R}^{n} \mathbf{M}_{0} .
$$

A Binet-like formula for the generalized Mersenne quaternions is given in the following theorem.

Theorem 2.3 (Binet-like formula). For any nonnegative integer $n$, the $n$-th generalized Mersenne quaternion is

$$
\mathcal{M}_{n}^{(p, r, s)}=2^{n} \underline{\boldsymbol{M}}^{(p, r, s)}-\underline{\boldsymbol{A}}
$$

where $\underline{\boldsymbol{M}}^{(p, r, s)}=1+i 2^{p}+j 2^{r}+k 2^{s}$. 
Proof. Using Eq. (2) and Definition 2.1, we can write

$$
\begin{aligned}
\mathcal{M}_{n}^{(p, r, s)} & =M_{n}+i M_{n+p}+j M_{n+r}+k M_{n+s} \\
& =\left(2^{n}-1\right)+i\left(2^{n+p}-1\right)+j\left(2^{n+r}-1\right)+k\left(2^{n+s}-1\right) \\
& =2^{n}\left(1+i 2^{p}+j 2^{r}+k 2^{s}\right)-(1+i+j+k) .
\end{aligned}
$$

The last equation gives the theorem.

The following lemma gives the generalized Mersenne quaternions with negative indices.

Theorem 2.4. For any integer $n$, we have

$$
\mathcal{M}_{-n}^{(p, r, s)}=-\frac{1}{2^{n}}\left(\mathcal{M}_{n}^{(-p,-r,-s)} \circ \underline{\boldsymbol{M}}^{(p, r, s)}\right),
$$

where the operator "०” is the point-wise product of two quaternions given by

$$
\begin{aligned}
q^{(1)} \circ q^{(2)} & =\left(q_{0}^{(1)}+i q_{1}^{(1)}+j q_{2}^{(1)}+k q_{3}^{(1)}\right) \circ\left(q_{0}^{(2)}+i q_{1}^{(2)}+j q_{2}^{(2)}+k q_{3}^{(2)}\right) \\
& =q_{0}^{(1)} q_{0}^{(2)}+i q_{1}^{(1)} q_{1}^{(2)}+j q_{2}^{(1)} q_{2}^{(2)}+k q_{3}^{(1)} q_{3}^{(2)} .
\end{aligned}
$$

Proof. From Eqs. (4) and (5), we obtain

$$
\mathcal{M}_{n-1}^{(p, r, s)}=\frac{1}{2}\left(3 \mathcal{M}_{n}^{(p, r, s)}-\mathcal{M}_{n+1}^{(p, r, s)}\right)=\frac{1}{2}\left(\mathcal{M}_{n}^{(p, r, s)}-\underline{\mathbf{A}}\right) .
$$

Using the last equation, we have

$$
\begin{aligned}
\mathcal{M}_{-n}^{(p, r, s)} & =M_{-n}+i M_{-n+p}+j M_{-n+r}+k M_{-n+s} \\
& =-\frac{M_{n}}{2^{n}}-i \frac{M_{n-p}}{2^{n-p}}-j \frac{M_{n-r}}{2^{n-r}}-k \frac{M_{n-s}}{2^{n-s}} \\
& =-\frac{1}{2^{n}}\left(M_{n}+i 2^{p} M_{n-p}+j 2^{r} M_{n-r}+k 2^{s} M_{n-s}\right) .
\end{aligned}
$$

The result follows from the last equation.

The following theorem gives the norm of a generalized Mersenne quaternion.

Theorem 2.5. For any integer $n$, we have

$$
N\left(\mathcal{M}_{n}^{(p, r, s)}\right)=2^{2 n} N\left(\underline{\boldsymbol{M}}^{(p, r, s)}\right)-2^{n+1} \Omega^{(p, r, s)}+4,
$$

where $\Omega^{(p, r, s)}=1+2^{p}+2^{r}+2^{s}$.

Proof. From Eqs. (1) and (2), we have

$$
\begin{aligned}
N\left(\mathcal{M}_{n}^{(p, r, s)}\right) & =\left(2^{n}-1\right)^{2}+\left(2^{n+p}-1\right)^{2}+\left(2^{n+r}-1\right)^{2}+\left(2^{n+s}-1\right)^{2} \\
& =2^{2 n}\left(1+2^{2 p}+2^{2 r}+2^{2 s}\right)-2^{n+1}\left(1+2^{p}+2^{r}+2^{s}\right)+4
\end{aligned}
$$

and this equation gives the theorem.

Now we give some summation formulas for the generalized Mersenne quaternions. 
Theorem 2.6. For any nonnegative integer $n$, we have

$$
\sum_{v=0}^{n} \mathcal{M}_{v}^{(p, r, s)}=\mathcal{M}_{n+1}^{(p, r, s)}-\underline{\boldsymbol{M}}^{(p, r, s)}-n \underline{\boldsymbol{A}}
$$

and

$$
\sum_{v=0}^{n}\left(\begin{array}{l}
n \\
v
\end{array}\right) \mathcal{M}_{v}^{(p, r, s)}=\left(\frac{3}{2}\right)^{n}\left(\mathcal{M}_{n}^{(p, r, s)}+\left[1-\left(\frac{4}{3}\right)^{n}\right] \underline{\boldsymbol{A}}\right)
$$

Proof. Let us consider $a_{v}=\mathcal{M}_{v+1}^{(p, r, s)}-\underline{\mathbf{M}}^{(p, r, s)}-v \underline{\mathbf{A}}$. Hence, by Eq. (5), we obtain

$$
a_{v}-a_{v-1}=\mathcal{M}_{v}^{(p, r, s)} .
$$

Applying the idea of "creative telescoping" in [9] to Eq. (9), we conclude

$$
\begin{aligned}
\sum_{v=0}^{n} \mathcal{M}_{v}^{(p, r, s)}=\sum_{v=0}^{n}\left(a_{v}-a_{v-1}\right) & =a_{n}-a_{-1} \\
& =\mathcal{M}_{n+1}^{(p, r, s)}-\underline{\mathbf{M}}^{(p, r, s)}-n \underline{\mathbf{A}}-\mathcal{M}_{0}^{(p, r, s)}+\underline{\mathbf{M}}^{(p, r, s)}-\underline{\mathbf{A}} \\
& =\mathcal{M}_{n+1}^{(p, r, s)}-\mathcal{M}_{0}^{(p, r, s)}-(n+1) \underline{\mathbf{A}} \\
& =\mathcal{M}_{n+1}^{(p, r, s)}-\left(\underline{\mathbf{M}}^{(p, r, s)}-\underline{\mathbf{A}}\right)-(n+1) \underline{\mathbf{A}} \\
& =\mathcal{M}_{n+1}^{(p, r, s)}-\underline{\mathbf{M}}^{(p, r, s)}-n \underline{\mathbf{A}},
\end{aligned}
$$

which is the desired result.

From Theorem 2.3, we have

$$
\begin{aligned}
\sum_{v=0}^{n}\left(\begin{array}{l}
n \\
v
\end{array}\right) \mathcal{M}_{v}^{(p, r, s)} & =\sum_{v=0}^{n}\left(\begin{array}{l}
n \\
v
\end{array}\right)\left(2^{v} \underline{\mathbf{M}}^{(p, r, s)}-\underline{\mathbf{A}}\right) \\
& =\underline{\mathbf{M}}^{(p, r, s)} \sum_{v=0}^{n}\left(\begin{array}{l}
n \\
v
\end{array}\right) 2^{v}-\underline{\mathbf{A}} \sum_{v=0}^{n}\left(\begin{array}{l}
n \\
v
\end{array}\right) \\
& =\underline{\mathbf{M}}^{(p, r, s)} 3^{n}-\underline{\mathbf{A}} 2^{n} .
\end{aligned}
$$

Some elementary operations give Eq.(10).

A generating function for the generalized Mersenne quaternions can be found in the following theorem.

Theorem 2.7 (Generating function). A generating function for the generalized Mersenne quaternions is

$$
\sum_{v=0}^{\infty} \mathcal{M}_{v}^{(p, r, s)} t^{v}=\frac{\mathcal{M}_{0}^{(p, r, s)}-\left(\mathcal{M}_{1}^{(p, r, s)}-\underline{\boldsymbol{A}}\right) t}{1-3 t+2 t^{2}}
$$

Proof. Let us define

$$
G(x, t)=\sum_{v=0}^{\infty} \mathcal{M}_{v}^{(p, r, s)} t^{v}
$$


Multiplying this equation by $1,-3 t$, and $2 t^{2}$, respectively, and summing the last equations, we obtain

$$
\begin{aligned}
\left(1-3 t+2 t^{2}\right) G(x, t) & =\mathcal{M}_{0}^{(p, r, s)}+\left(\mathcal{M}_{1}^{(p, r, s)}-3 \mathcal{M}_{0}^{(p, r, s)}\right) t \\
& +\sum_{v=0}^{\infty}\left(\mathcal{M}_{n+2}^{(p, r, s)}-3 \mathcal{M}_{n+1}^{(p, r, s)}+2 \mathcal{M}_{n}^{(p, r, s)}\right) t^{n+2} \\
& =\mathcal{M}_{0}^{(p, r, s)}-2 \mathcal{M}_{-1}^{(p, r, s)} t .
\end{aligned}
$$

Also we have

$$
\begin{aligned}
2 \mathcal{M}_{-1}^{(p, r, s)} & =2 M_{-1}+2 i M_{p-1}+2 j M_{r-1}+2 k M_{s-1} \\
& =-M_{1}+i\left(M_{p}-1\right)+j\left(M_{r}-1\right)+k\left(M_{s}-1\right) \\
& =1+i 2^{p}+j 2^{r}+k 2^{s}-2(1+i+j+k) \\
& =\underline{\mathbf{M}}^{(p, r, s)}-2 \underline{\mathbf{A}} .
\end{aligned}
$$

Eqs. (11) and (12) give the theorem.

We give Catalan's identity for the generalized Mersenne quaternions. We calculate this identity by using the multiplication rules for quaternions.

Theorem 2.8 (Catalan's identity). Let $n$ and $m$ be any integers. Hence, we have

$$
\begin{aligned}
\mathcal{M}_{n-m}^{(p, r, s)} \mathcal{M}_{n+m}^{(p, r, s)}-\left[\mathcal{M}_{n}^{(p, r, s)}\right]^{2}=2^{n-m} & M_{m}^{2}\left(\Omega^{(p, r, s)} \underline{\boldsymbol{A}}^{*}-2\right) \\
& +2^{n+1}\left[M_{m}\left(\underline{\boldsymbol{M}}^{(r, s, p)}-1\right)+M_{-m}\left(\underline{\boldsymbol{M}}^{(s, p, r)}-1\right)\right] .
\end{aligned}
$$

Proof. We should calculate the coefficients of $1, i, j$, and $k$, separately. We only show the real part in detail. Hence, we can calculate it as

$$
\begin{aligned}
& \left(2^{n-m}-1\right)\left(2^{n+m}-1\right)-\left(2^{n-m+p}-1\right)\left(2^{n+m+p}-1\right)-\left(2^{n-m+r}-1\right)\left(2^{n+m+r}-1\right) \\
& \quad-\left(2^{n-m+s}-1\right)\left(2^{n+m+s}-1\right)+\left(2^{n+s}-1\right)^{2}-\left(2^{n}-1\right)^{2}+\left(2^{n+p}-1\right)^{2}+\left(2^{n+r}-1\right)^{2} \\
& =\left(2^{2 n}-2^{n-m}-2^{n+m}+1\right)+\left(-2^{2(n+p)}+2^{n-m+p}+2^{n+m+p}-1\right) \\
& \quad+\left(-2^{2(n+r)}+2^{n-m+r}+2^{n+m+r}-1\right)+\left(-2^{2(n+s)}+2^{n-m+s}+2^{n+m+s}-1\right) \\
& \quad+\left(2^{2(n+s)}-2^{n+s+1}+1\right)+\left(2^{2(n+r)}-2^{n+r+1}+1\right) \\
& \quad+\left(2^{2(n+p)}-2^{n+p+1}+1\right)+\left(-2^{2 n}+2^{n+1}-1\right) \\
& \left.\quad+2^{r}+2^{s}\right)+2^{n+m}\left(-1+2^{p}+2^{r}+2^{s}\right)-2^{n+1}\left(-1+2^{p}+2^{r}+2^{s}\right) .
\end{aligned}
$$

Consequently, we obtain the real part of the Catalan's identity for the generalized Mersenne quaternions in the form

$$
\left(2^{n+m}+2^{n-m}-2^{n+1}\right)\left(-1+2^{p}+2^{r}+2^{s}\right) .
$$

Similarly, the coefficients of $i, j$, and $k$ are

$$
\left(2^{n+1}-2^{n+m}-2^{n-m}\right)\left(1+2^{p}+2^{r}+2^{s}\right)+2^{n+1}\left(2^{m+r}+2^{-m+s}-2^{r}-2^{s}\right),
$$




$$
\left(2^{n+1}-2^{n+m}-2^{n-m}\right)\left(1+2^{p}+2^{r}+2^{s}\right)+2^{n+1}\left(2^{-m+p}+2^{m+s}-2^{p}-2^{s}\right)
$$

and

$$
\left(2^{n+1}-2^{n+m}-2^{n-m}\right)\left(1+2^{p}+2^{r}+2^{s}\right)+2^{n+1}\left(2^{m+p}+2^{-m+r}-2^{p}-2^{r}\right),
$$

respectively. After some elementary operations on the coefficients of $1, i, j$, and $k$, we obtain the theorem.

For $m=1$, the Catalan's identity gives the famous Cassini-type formula, which can be found in the next theorem.

Corollary 2.8.1 (Cassini's identity). For any integer $n$, we have

$$
\mathcal{M}_{n-1}^{(p, r, s)} \mathcal{M}_{n+1}^{(p, r, s)}-\left[\mathcal{M}_{n}^{(p, r, s)}\right]^{2}=2^{n-1}\left(\Omega^{(p, r, s)} \underline{\boldsymbol{A}}^{*}-2\right)+2^{n+1}\left(\underline{\boldsymbol{M}}^{(r, s, p)}-\underline{\boldsymbol{M}}^{(s, p, r)}\right) .
$$

The next theorem gives the d'Ocagne's identity for the generalized Mersenne quaternions.

Theorem 2.9 (d'Ocagne's identity). For any integers $m$ and $n$, we have

$$
\mathcal{M}_{m}^{(p, r, s)} \mathcal{M}_{n+1}^{(p, r, s)}-\mathcal{M}_{m+1}^{(p, r, s)} \mathcal{M}_{n}^{(p, r, s)}=2^{m+1}\left[\underline{\boldsymbol{M}}^{(s, p, r)}-\left(2^{m}-2^{n}\right) \Omega^{(p, r, s)} \underline{\boldsymbol{A}}-2^{n+1} \underline{\boldsymbol{M}}^{(r, s, p)}\right]^{*} .
$$

Proof. We calculate the coefficients of $1, i, j$, and $k$ in a similar way as the proof of Catalan's identity, respectively. The real part is

$$
\begin{gathered}
\left(2^{m}-1\right)\left(2^{n+1}-1\right)-\left(2^{m+p}-1\right)\left(2^{n+p+1}-1\right)-\left(2^{m+r}-1\right)\left(2^{n+r+1}-1\right) \\
-\left(2^{m+s}-1\right)\left(2^{n+s+1}-1\right)+\left(2^{m+s+1}-1\right)\left(2^{n+s}-1\right)-\left(2^{m+1}-1\right)\left(2^{n}-1\right) \\
\quad+\left(2^{m+p+1}-1\right)\left(2^{n+p}-1\right)+\left(2^{m+r+1}-1\right)\left(2^{n+r}-1\right) \\
=-2^{n+1}+2^{n+p+1}+2^{n+r+1}+2^{n+s+1} 2^{m+1}-2^{m+p+1}-2^{m+r+1} \\
\quad-2^{m+s+1}-2^{m}+2^{m+p}+2^{m+r}+2^{m+s}+2^{n}-2^{n+p}-2^{n+r}-2^{n+s} \\
=\left(2^{m+1}-2^{n+1}-2^{m}+2^{n}\right)\left(1-2^{p}-2^{r}-2^{s}\right) \\
=\left(2^{m}-2^{n}\right)\left(1-2^{p}-2^{r}-2^{s}\right) .
\end{gathered}
$$

Similarly, the coefficients of $i, j$, and $k$ are

$$
\begin{aligned}
& 2^{m}\left(1+2^{p}+2^{r}-2^{s}\right)-2^{n}\left(1+2^{p}-2^{r}+2^{s}\right), \\
& 2^{m}\left(1-2^{p}+2^{r}+2^{s}\right)-2^{n}\left(1+2^{p}+2^{r}-2^{s}\right),
\end{aligned}
$$

and

$$
2^{m}\left(1+2^{p}-2^{r}+2^{s}\right)-2^{n}\left(1-2^{p}+2^{r}+2^{s}\right),
$$

respectively. If we consider the coefficients $i, j, k$, and the real part together, we have

$$
\begin{array}{r}
\mathcal{M}_{m}^{(p, r, s)} \mathcal{M}_{n+1}^{(p, r, s)}-\mathcal{M}_{m+1}^{(p, r, s)} \mathcal{M}_{n}^{(p, r, s)}=\left(2^{m}-2^{n}\right)\left(1-2^{p}-2^{r}-2^{s}\right)(1+i+j+k) \\
+2^{m+1}\left(1+i 2^{p}+j 2^{r}+k 2^{s}+1+i 2^{r}+j 2^{s}+k 2^{p}-2\right) \\
-2^{n+1}\left(1+i 2^{p}+j 2^{r}+k 2^{s}+1+i 2^{s}+j 2^{p}+k 2^{r}-2\right) \\
=\left(2^{m}-2^{n}\right)\left(1-2^{p}-2^{r}-2^{s}\right) \underline{A}+2^{m+1}\left(\underline{\mathbf{M}}^{(p, r, s)}+\underline{\mathbf{M}}^{(r, s, p)}-2\right) \\
-2^{n+1}\left(\underline{\mathbf{M}}^{(p, r, s)}+\underline{\mathbf{M}}^{(s, r, p)}-2\right) .
\end{array}
$$

With some elementary operations in the last equation, we obtain the theorem. 


\section{Appendix}

We mentioned above that Mersenne quaternions can be defined by taking $p=1, r=2$, and $s=3$. In this section, we calculate some properties of Mersenne quaternions. Let $\tilde{M}_{n}$ be the $n$-th Mersenne quaternion. We have

$$
\begin{gathered}
\tilde{\mathcal{M}}_{n}=M_{n}+i M_{n+1}+j M_{n+2}+k M_{n+3}, \\
\tilde{\mathcal{M}}_{n+1}=2 \tilde{\mathcal{M}}_{n}+\underline{\mathbf{A}}, \\
\tilde{\mathcal{M}}_{n+1}=3 \tilde{\mathcal{M}}_{n}-2 \tilde{\mathcal{M}}_{n-1}, \\
\tilde{\mathcal{M}}_{n}=2^{n} \tilde{\mathbf{M}}-\underline{\mathbf{A}} \text { where } \tilde{\mathbf{M}}=1+2 i+4 j+8 k, \\
{\left[N\left(\tilde{\mathcal{M}}_{n}\right)\right]^{2}=85.2^{2 n}-15.2^{n+1}+4,} \\
\sum_{v=0}^{n} \tilde{\mathcal{M}}_{v}=\tilde{\mathcal{M}}_{n+1}-\tilde{\mathbf{M}}-n \underline{\mathbf{A}},
\end{gathered}
$$

and

$$
\sum_{v=0}^{n}\left(\begin{array}{l}
n \\
v
\end{array}\right) \tilde{\mathcal{M}}_{v}=\left(\frac{3}{2}\right)^{n}\left(\tilde{\mathbf{M}}+\left[1-\left(\frac{4}{3}\right)^{n}\right] \underline{\mathbf{A}}\right) .
$$

\section{Acknowledgements}

Research on this paper was supported by the Research Fund of Kastamonu University via project number KÜBAP-01/2017-1. The authors also wish to thank the anonymous referees for their suggestions that led to improving our paper.

\section{References}

[1] Berzsenyi, G. (1977). Gaussian Fibonacci numbers, Fibonacci Quart., 15, 233-236.

[2] Catarino, P., Campos, H., \& Vasco, P. (2016). On the Mersenne sequence, Ann. Math. Inform., 46, 37-53.

[3] Cimen, C. B., \& Ipek, A. (2016). On Pell quaternions and Pell-Lucas quaternions, Adv. Appl. Clifford Algebr., 26, 39-51.

[4] Hamilton, W. R. (1853). Lectures on Quaternions, Hodges and Smith, Dublin.

[5] Horadam, A. F. (1963). Complex Fibonacci numbers and Fibonacci quaternions, Amer. Math. Monthly., 70, 289-291.

[6] Szynal-Liana, A., \& Wloch, I. (2016). The Pell quaternions and the Pell octonions, Adv. Appl. Clifford Algebr., 26, 435-440. 
[7] Szynal-Liana, A., \& Wloch, I. (2016). A note on Jacobsthal quaternions, Adv. Appl. Clifford Algebr., 26, 441-447.

[8] Wright, G. H., \& Hardy, E. M. (1975). An Introduction to the Theory of Numbers, Oxford University Press, Oxford.

[9] Zeilberger, D. (1991). The method of creative telescoping, J. Symbolic Comput., 11, 195-204. 